\title{
SOME POINTS IN THE MODERN TECHNIQUE OF CATARACT EXTRACTION.
}

\author{
$B y$ H. B. STALLARD, M.D., F.R.C.S.
}

(Assistant Ophthalmic Surgeon, St. Bartholomew's Hospital. Assistant Surgeon, Moorfields Eye Hospital. Ophthalmic Surgeon, Mount Vernon Hospital.)

Ophthalmic surgeons in the past used to advise those they instructed to perform the cataract extraction operation with speed, compatible with efficiency, and with the minimum interference to the patient. To a great extent this advice still holds good so far as the actual operation of extraction of the cataractous lens is concerned, but in other features the technique of preparing for the operation and post-operative care has changed vastly. To-day the preparation of the patient is directed towards the elimination of hazards both during and after operation by rendering him unable to endanger its success, in other words to fulfil the ideal of modern surgery by making the operation as safe as possible for the patient.

In this short article I do not intend to cover all the ground concerning preoperative preparation, operative technique and post-operative treatment, but rather to write about certain points which I have found work with advantage in practice and which I consider valuable to include in the technique which is most suited to me.

\section{Anæsthesia and Akinesia.}

I prefer to use 4-6 drops of pantocain I per cent. and 2 drops of adrenalin I/ Iooo. Pantocain is a novocain derivative, it does not damage the corneal epithelium nor cause coffee-ground vomiting after operation as cocaine sometimes does, and so far I have never seen any toxic manifestations after its use in several hundred cases. It acts more quickly than cocaine and I think it also has an advantage in that patients do not feel the weight of instruments touching the eye as happens with other local anæsthetics. It does not raise the intra-ocular pressure and its only disadvantage is the slight hyperæmia it induces after instillation in some persons and its failure to check capillary oozing as cocaine does, but these defects are readily overcome by instilling adrenalin drops after the pantocain.

Facial Nerve Block. Anæsthesia and akinesia of the orbicularis muscle may be effected by the injection of novocain 2 per cent. through the skin and down to the periosteum of the malar bone $i \mathrm{~cm}$. below and posterior to the lower and outer angle of the orbit. The injection is carried vertically upwards to a point $I \mathrm{~cm}$. above the supra-orbital margin and horizontally below the infra-orbital margin to the infra-orbital foramen, and if necessary horizontally along the supraorbital margin to the supra-orbital notch. This method has the advantage of producing both akinesia and anæsthesia of the entire operation area. Its disadvantage is that some swelling of the lids may cause some mechanical embarrassment and that akinesia is not always so complete as it is after an injection of the upper branches of the facial nerve as they cross the neck of the mandible. (O'Brien's method). However, akinesia of the orbicularis is an essential safeguard against the patient damaging his eye by violent contraction of this muscle.

Retro-ocular Injection. An injection of 1.5 cc. of 4 per cent. novocain into Tenon's capsule prevents any pain that may arise from touching the iris or other intra-ocular manœuvres that may be necessary. Some surgeons prefer a retroocular injection of I cc. of novocain into the ciliary ganglion, but this risks an orbital hæmorrhage and lowers the intra-ocular pressure almost immediately. I think the Tenon's capsule injection is safer and quite effective. It is advisable to wait about 7 minutes for these injections to work. 


\section{Dilatation of the Pupil.}

Good dilatation of the pupil before operation is essential. Five or six applications of homatropine I per cent. at half hourly intervals before operation is generally sufficient, but this may have to be augmented by the use of homatropine lamallæ or a subconjunctival injection of 2 or 3 minims of adrenalin chloride I/ 1000 at 6 o'clock $3 \mathrm{~mm}$. from the limbus at a $\frac{1}{4}$ to $\frac{1}{2}$ hour before operation.

\section{Retractors.}

In some instances it is possible to dispense with retractors altogether, the lids being held apart by No. I silk sutures passed transversely through the skin of the upper and lower lids $3 \mathrm{~mm}$. behind the centre of the lash margin and clamped to the towels of the head and the face with Spencer Wells forceps.

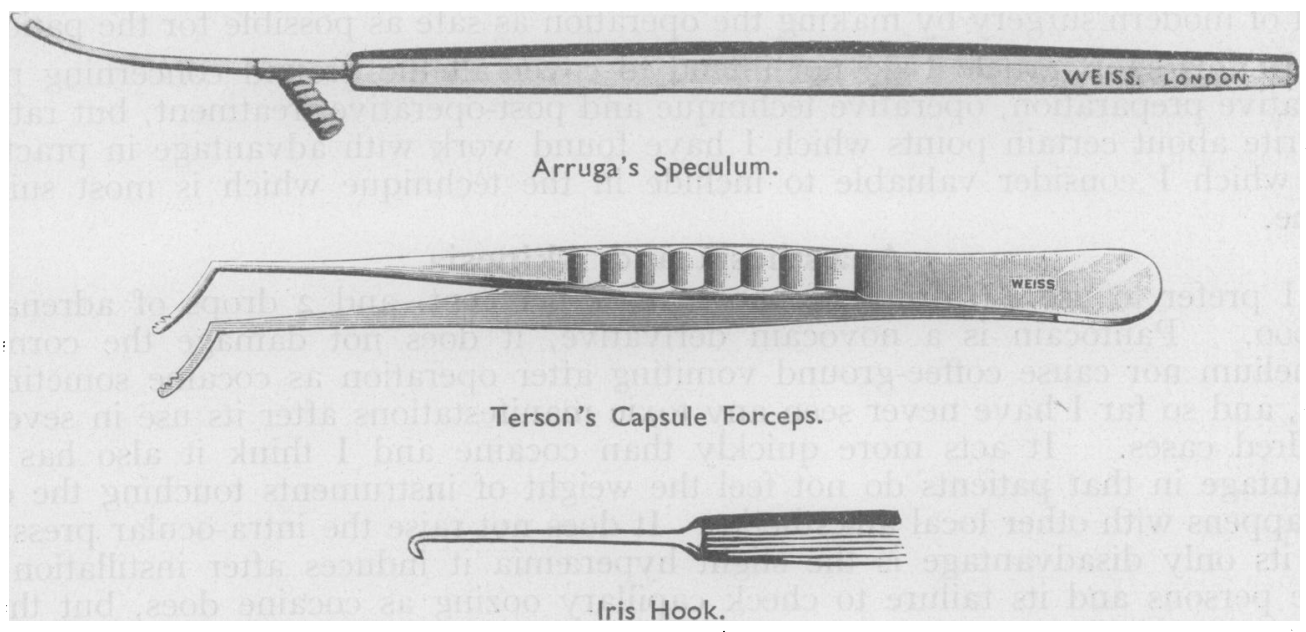

FIG. 1 .

Arruga's speculum (see Fig. I) which is constructed so that the weight of the retractor is borne on the bridge of the nose and the skin in the temporal region removes any source of danger from the retractor pressing on the eye. I prefer to use this retractor made with a dull black finish so that light reflexes from its edges are eliminated.

\section{The Superior Rectus Muscle.}

If it is evident from a previous examination that the patient will not look down when directed to do so or is unco-operative in the matter of keeping his eye reasonably still, some control may be obtained either by injecting the belly of the superior rectus muscle with I cc. of novocain or by the insertion of a No. I silk suture passed through its tendon. Some surgeons prefer to tie this suture to a small projection on the upper shank of the speculum, others like to leave it held by an assistant. This stitch is not altogether free from inflicting damage for if the patient looks down too far the sclera is dragged upon, the wound opens widely and vitreous may escape. I think it is safer to inject I cc. of novocain 2 per cent. into the belly of the superior rectus muscle and to wait 7 minutes for this to work before beginning the operation. 


\section{Corneo-scleral Stitch.}

I have found that a corneo-scleral stitch is a most valuable addition to the surgical technique of cataract extraction, whether the intra-capsular or the extracapsular operation is performed.

Its chief advantages are that the wound is readily controlled. In intracapsular extractions, in high myopes with fluid degenerate vitreous and other cases in which vitreous loss may be feared the immediate tightening and tying of this suture is the only procedure which will check a relentless forward flow of vitreous.

The stitch when tied so that the wound edges are firmly coapted gives considerable security against post-operative hyphæma. In 72 consecutive and unselected cases of extra-capsular extraction and 23 cases of intra-capsular extraction which I have recently performed there has been no case of post-operative hyphæma. I believe that the average incidence of post-operative hyphæma of all degrees is 25 to 30 per cent. and sometimes it is higher. On this account alone the insertion of this stitch is worth while. A hyphæma prolongs convalescence, it may endanger the sight by causing occlusio pupillæ, blood staining of the cornea and secondary glaucoma in severe cases and in milder degrees it may irritate the eye for weeks.

The liability to iris prolapse is almost negligible after a properly applied corneoscleral stitch. In 2 cases a thin strip of iris about $0.5 \mathrm{~mm}$. by $\mathrm{I} \mathrm{mm}$. presented in the deeper part of the wound, in one of these on the temporal side and in the other on the nasal side. In both these cases I was conscious of the fact that in tying the corneo-scleral suture I had not coapted to the margins of the section wound with that degree of firmness which is desirable. Both cases had a somewhat atonic iris and it was probably tempting Providence not to have done a peripheral iridectomy. Replacement of the iris or iridectomy may be quite readily effected with the stitch in place.

Another advantage is that the scleral part of the stitch may be held in plain forceps and so used for fixation of the globe in old persons in whom the conjunctiva is very thin and liable to tear with fixation forceps. The corneal part of the stitch held in plain forceps may be used to lift the corneal edge of the section wound forwards and so facilitate the introduction of capsule forceps.

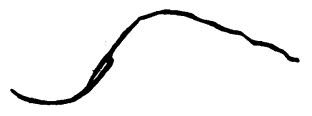

$\nabla$

FIG. 2.

Eyeless needle for passing corneo-scleral stitch. Diagram shows a section of the body of the needle which is triangular in shape with the cutting spine on the outer convex curve.

The needle (see Fig. 2) was made for me by the Mersuture Co. The spine is on the outer convex surface of the needle the concavity being flat. The lateral edges and summit of the spine cut. The needle ends in a rounded shaft which is , eyeless and contains a ooo silk suture. The spine on the convex aspect splits the tissues deeply. A spine on the concave aspect, as in the Maddox needles, is liable to split the tissues superficially and to tear out the suture.

The needle is held in a Quarry Silcock's needle holder with fine jaws. 
It is sometimes better to pass it before the speculum is inserted as the upper shank of the Arruga's speculum prevents the accurate application of the needle.

To steady the globe the insertion of the internal rectus muscle is fixed with toothed forceps for the right eye and the external rectus muscle for the left eye. Fixation of the rectus muscle is maintained until the stitch is completed.

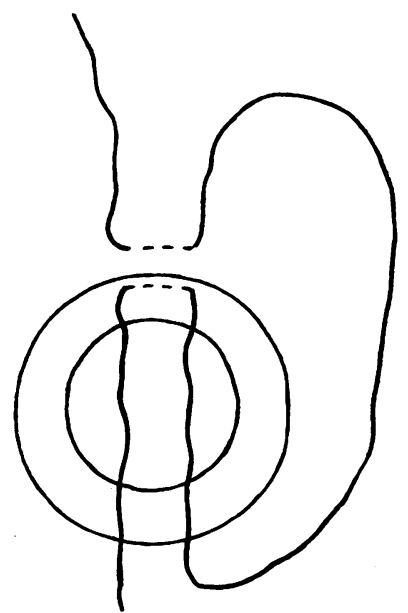

FIG. 3.

Diagram of the site of the corneo-scleral stitch and the manner in which the ends are placed before section is made with the cataract knife.

The needle is then passed transversely through half the thickness of the substantia propria I mm. inside the limbus and for a distance of $2 \mathrm{~mm}$., it is then carried through the conjunctiva, episcleral tissue and half the thickness of the sclera I.5. mm. behind the limbus and opposite to and parallel with the corneal part of the suture (see Fig. 3).

The loop end and free end of the corneal stitch are placed vertically over the cornea to the $60^{\prime}$ clock meridian and the scleral ends are placed upwards in the I2 o'clock meridian. The limbus is then clear for the cataract section (see Fig. 3).

When inserting the corneo-scleral stitch I have found it helpful to use operating glasses. A mild degree of striate keratitis localized in the upper third of the cornea has been the only complication from using a corneo-scleral stitch. This generally clears up in 8-1o days and leaves no ill effect. It is not so severe as the striate keratitis which sometimes occurs after cataract extraction when a corneoscleral stitch has not been used.

The amount of astigmatism after using this stitch has been on an average less than in cases where I have in the past not used the stitch. In some cases there was no astigmatism and the average has been between $I \cdot 5$ and $2 \cdot 25 \mathrm{D}$, axis $0^{\circ}$ to $30^{\circ}$.

\section{Capsule Forceps}

In the extra-capsular extraction I think it is best to remove as much of the anterior capsule as possible. This avoids the formation of a dense " aftercataract " with thick bands crossing the pupil. It may be effected by capsule forceps which have short curved shanks to pass over and clear the iris before the straight teeth bearing terminals are reached (see Fig. I). The teeth may be on an average 4 into 5 and when locked their under surface should be smooth so that the iris is not damaged in passing the closed instrument into the eye. The capsule 
forceps are passed in the anterior chamber to a point I or $2 \mathrm{~mm}$. below the centre of the pupil and are opened as wide as the degree of pupillary dilatation will allow, the teeth are pressed gently into the capsule and the forceps closed. The instrument is at first moved to and fro laterally then downwards and finally upwards and out of the wound, a large central hole is thus torn in the anterior capsule. Only about 7 per cent. of cases where a good opening has been made require capsulotomy.

A blunt hook (see Fig. I) is an invaluable instrument in some cases of cataract extraction where the pupil is small and the surgeon wishes to avoid the mutilation of an iridectomy. When the upper pole of the lens is tilted forwards with the iris stretched over it the blunt hook is slipped round the pupil margin and by gentle traction the iris is drawn towards the ciliary body at I2 o'clock until the equator of the lens presents; its delivery is then completed by pressure from below and by turning the hook so that it engages in the posterior cortex of the lens and assists in wheeling it out of the wound.

In cases where the iris bulges up into the wound after the section is completed and it is feared that the suspensory ligament has undergone spontaneous rupture and the vitreous face is presenting beneath the iris, it is clear that any pressure on the eye in delivering the lens may precipitate a vitreous loss. In such cases the upper pole of the lens may be tilted very gently forwards by pressure over the lower pole with a curette or strabismus hook and as soon as the upper pole appears it is incised with a cystitome the blade of which may then be introduced on the flat behind the upper pole and then turned forwards so that its point transfixes the posterior cortex of the lens.

In this manner the lens may be picked out of the eye with the minimum pressure having been applied to the globe. Immediately the lens is out of the wound the corneo-scleral stitch is drawn together and tied. Toilet of the iris and if necessary peripheral iridectomy can be effected after the stitch is tied.

\section{Anterior Chamber Wash-out.}

A careful anterior chamber wash-out is necessary in cases of immature cataract in order to avoid the dangers of iritis from irritation of the soft lens matter and even destruction of the eye from endophthalmitis phako-anaphylactica. Rycroft's irrigator is particularly good for this work. It is flat and easily introduced into the eye and will also act as an iris repositor. A prolonged anterior chamber wash-out is undesirable. Soft lens matters may be gently pressed from under the iris towards the pupil by intermittent stroking pressure on the periphery of the cornea with an iris repositor at suitable sites.

\section{Intra-capsular Cataract Extraction.}

In some selected cases it seems more desirable to extract the lens in its capsule. When this is effected without mishap during operation the results are admirable. After operation the eye shows very little evidence of disturbance, there is often no sign of ciliary vascular injection as there is after most cases of extra-capsular extraction, and other complications due to the presence of lens matter and capsule such as iritis, cyclitis, raised intra-ocular pressure and occlusio pupillæ do not occur. It has been suggested that glaucoma and retinal detachment may be complications of the intra-capsular operation but from a review of such statistics as are available it does not seem that these disasters are any more likely to follow the intra-capsular than the extra-capsular operation for they are known to occur after both types of operation. However, before any definite statements can be 
made on this point it will be necessary to make a thorough survey and long follow-up of a larger collection of material than has hitherto been available.

The intra-capsular operation is particularly favourable for immature cataract $\overrightarrow{\vec{F}}$ in persons over 50, but is contraindicated in the very elderly, in high myopes, in $\stackrel{0}{\circ}$ cases where the vitreous is known to be degenerate, glaucoma, hyperpiesia and in some cases of cataract complicated by iridocyclitis. Under 50 years of age it is probable that the suspensory ligament may prove more resistant than the anterior capsule and so the capsule is liable to tear before the lens is dislocated, a matter which leads to no unpleasant consequences provided the suspensory ligament has on not been partly torn and the vitreous displaced forwards.

Some surgeons state that cataract complicated by the results of iritis or cyclitis which have long since subsided should not be extracted by the intra-capsular method whilst others are in favour of doing so. The impression I have gained from my own limited experience of these cases is that after the extra-capsular extraction the pupil is sometimes liable to occlusion from dense capsular remains and fibrous tissue the contraction of which draws its margin to the centre, neces- $\vec{\infty}$ sitating often a number of capsulotomies and iridotomies and severe cases ultimately require removal of a piece of iris and capsule to reconstruct an adequate pupillary aperture. In cases where the iritis has not been severe and the intra-ocular pressure is normal $I$ have done a preliminary iridectomy in order to gain some impression as to the manner in which the eye will react to surgical attack and also to obtain an idea about the nature and disposition of the posterior synechiæ. If the latter are limited to the pupillary region and are not extensive and if the eye responds favourably to iridectomy I have preferred to extract the lens by the intra-capsulas $\overrightarrow{0}$ method. After breaking through the posterior synechiæ with a few gentle sweeps. of an iris repositor the capsule forceps are applied to the lens in the I2 o'cloc meridian just in front of the equator (the preliminary iridectomy renders this site for application readily exposed) the forceps are separated $3 \mathrm{~mm}$. and pressed gently into the capsule and then closed. Rotatory movements, clockwise and anticlockwise, at first small excursions and then larger are made in the plane of the $\triangle$ equator. When the upper pole of the lens is felt to come forward the lens is gently lifted forwards and upwards, the lateral movements are continued over a little wider excursion and simultaneously a crochet is placed over the lower part of the cornea below the lower pole of the lens. From this moment the capsule is not pulled on by the forceps but delivery is effected mainly by moving the crochet upwards over the cornea in a zig-zag manner below the lower pole of the lens. until it is safely delivered from the wound.

The risks in this mode of delivery are a little greater than those involved in the process of " tumbling " the lens for the suspensory ligament is first torn through above and vitreous may come forward near the section wound. In the "tumbling", procedure capsule is seized in the 6 o'clock meridian just in front of the equator and when the suspensory ligament is torn through and the lower pole of the lens has come forward it is delivered by turning it over so that the lower pole comes 응 uppermost and the posterior lens capsule faces anteriorly. After the lens is dislo- N cated and delivery begun the forceps are used merely to hold the lens and to exert $\underset{\omega}{\mathcal{N}}$ the minimum traction possible, delivery being mainly effected by the follow up 0 with the crochet.

Arruga's forceps are particularly good for intra-capsular extraction. There is no need for undue haste in dislocating the lens. Sometimes this has to be patiently undertaken, the whole process lasting 30 to 60 seconds before the lens is ready for: delivery. Some beginners are apt to rush the manceuvre. Actually with good 
anæsthesia and akinesia, helpful co-operation from the patient and with a speculum like Arruga's which does not press on the eye, there is little risk in taking 30 to 60 seconds to effect a satisfactory rupture of the suspensory ligament.

The technique of intra-capsular extraction differs from that which I have described earlier in this paper under the extra-capsular operation particularly in the following points:-(I) Full dilatation of the pupil is essential. This may be assisted by a sub-conjunctival injection of 3 or 4 minims of adrenalin chloride I / IOOO at 6 o'clock about $2-3 \mathrm{~mm}$. from the limbus $\frac{1}{4}$ to $\frac{1}{2}$ hour before operation. (2) The section wound is larger and should be nearly half the circumference of the cornea. (3) A peripheral iridectomy is performed before the capsule forceps are introduced to extract the lens. (4) If blood enters the anterior chamber before the capsule forceps have grasped the lens satisfactorily it is washed out, as its presence makes the surface of the lens capsule slippery and the forceps may fail to grip the capsule. Immediately the lens is delivered the wound is closed by tightening the corneo-scleral stitch and the iris and wound toilet are then completed. Eserine is instilled and the eyes tied up with pad and bandaged with a Foster Moore many-tailed bandage.

On the $4^{\text {th }}$ day dressings may be omitted, the eye being protected by a Cartella or aluminium shield. On the 8th day dark glasses are worn by day and the eye guarded with a shield at night. The corneo-scleral stitch is removed on the I4th day after operation under pantocain local anæsthesia. To do this I prefer to use a Desmarres' retractor to hold up the upper lid and to remove the stitch by holding it in plane iris forceps and cutting it with a pair of ground down blunt-ended de Weckers iris scissors. It is important to pull the ends of the suture laterally in the plane of their insertion in order to avoid damage to the cornea and the section wound.

\section{Conclusion.}

The purpose of this short paper has been to describe briefly some points in the modern technique of cataract surgery. My remarks are far from being comprehensive for there is an immense number of technical variations introduced by different operators. After rendering the operation as safe as possible for the patient by preventing all hazards likely to arise during it there should be the minimum instrumentation of the eye necessary to effect the desired result. 